\title{
El mezquite como ayuda ergogénica para atletas de alto rendimiento en deportes intermitentes. Revisión sistemática.
}

\author{
Mesquite as an ergogenic aid for high-performance athletes in intermittent sports. Systematic \\ review.
}

\author{
Joselyn Reséndiz-Trejo ${ }^{a}$, Esther Ramírez-Moreno ${ }^{b}$, José A. Ariza-Ortega $^{c}$, Araceli Ortíz-Polo ${ }^{d}$
}

\begin{abstract}
:
High-performance athletes are constantly subjected to demanding loads of physical effort, for which reason ergogenic aids are often used to improve energy production, sports performance, body composition and / or enhance physiological adaptations to training. There are several studies about supplementation with polyphenols in foods such as berries, citrus fruits and cocoa; where its positive effect on performance has been demonstrated. However, there are other resources that will have significant content of these molecular complexes; an example of this is mesquite. Extracts obtained from the husk, pods, pollen and leaves of mesquite plants have been found to possess important phenolic compounds. However, the bibliography and research on the use of this plant in sports is scarce, therefore, the objective of this review is to know the properties and benefits of mesquite, to evaluate its effect on the physiological phenomena raised in intermittent sports and its feasibility of use as an ergogenic aid.
\end{abstract}

A systematic review was carried out divided into three topics: 1) Mesquite ("Composition of mesquite AND Mexico", "Mesquite AND polyphenols", "Mesquite AND exercise"), 2) Polyphenols ("Polyphenols AND inflammation AND sport OR exercise", Ergogenic aids AND polyphenols") and 3) Exercise-induced inflammation ("Sport performance AND intermittent sport AND inflammation ", "Intermittent exercise AND inflammation AND muscle performance AND damage ") From which a total of 33 articles were obtained, and after a Second review 16 of them were identified and analyzed. It was concluded that mesquite is a potentially profitable natural resource to be used as an ergogenic aid; being mesocarp flour and mesquite seed which have an important content of polyphenols and antioxidant capacity; This composition favors the reduction of oxidative stress, the attenuation of muscle pain, and the reduction of post-exercise recovery time. In view of all the benefits attributed to the composition of mesquite, but the limited evidence of the effects of its consumption, this review reveals that more research is needed on its use in sports.

\section{Keywords:}

Mesquite, polyphenols, sport performance, exercise-induced inflammation.

\section{Resumen:}

Los atletas de alto rendimiento están constantemente sometidos a cargas demandantes de esfuerzo físico, por lo cual muchas veces se recurre al consumo de ayudas ergogénicas para mejorar la producción de energía, el rendimiento deportivo, la composición corporal y/ o potenciar las adaptaciones fisiológicas al entrenamiento. Se han realizado numerosos estudios acerca de la suplementación con polifenoles en alimentos como frutos rojos, cítricos y cocoa; en donde se ha demostrado su efecto positivo sobre el rendimiento. No obstante, existen otros recursos que poseen un significativo contenido de estos complejos moleculares; ejemplo de ello es el mezquite.

\footnotetext{
a Universidad Autónoma del Estado de Hidalgo, Instituto de Ciencias de la Salud, https://orcid.org/0000-0003-2226-2493, Email: re353254@uaeh.edu.mx

b Universidad Autónoma del Estado de Hidalgo, Instituto de Ciencias de la Salud, https://orcid.org/0000-0002-9928-8600, Email: esther_ramirez@uaeh.edu.mx

c Universidad Autónoma del Estado de Hidalgo, Instituto de Ciencias de la Salud, http://orcid.org/0000-0002-2163-4593,Email: jose190375@hotmail.com

d Autor de correspondencia, Universidad Autónoma del Estado de Hidalgo, Instituto de Ciencias de la Salud, https://orcid.org/0000-00015561-2221, Email: arapolo@ hotmail.com
} 
Se ha encontrado que los extractos obtenidos de cáscara, vainas, polen y hojas de plantas del mezquite, poseen compuestos fenólicos importantes. Sin embargo, la bibliografía e investigaciones del uso de esta planta en el ámbito deportivo es escasa, por lo que, el objetivo de esta revisión es conocer las propiedades y beneficios del mezquite, para evaluar su efecto sobre los fenómenos fisiológicos suscitados en deportes intermitentes y su factibilidad de uso como ayuda ergogénica.

Se realizó una revisión sistemática dividida en tres temáticas: 1) Mezquite ("Mesquite composition AND Mexico", "Mesquite AND polyphenols", "Mesquite AND exercise"), 2) Polifenoles ("Polyphenols AND inflammation AND sport OR exercise", "Ergogenic aids AND polyphenols") y 3) Inflamación inducida por ejercicio ("Sport performance AND intermittent sport AND inflammation", "Intermittent exercise AND muscle inflammation AND performance AND damage") De la cual se obtuvo un total de 33 artículos, y tras una segunda revisión se identificaron y analizaron 16 de ellos. Se concluyó que el mezquite es un recurso natural potencialmente rentable para ser utilizado como ayuda ergogénica; siendo harina de mesocarpio y la semilla del mezquite las que poseen un importante contenido de polifenoles y capacidad antioxidante; dicha composición favorece la reducción del estrés oxidativo, la atenuación del dolor muscular, y la disminución del tiempo de recuperación post ejercicio. En vista de todos los beneficios atribuidos a la composición del mezquite, pero a la escasa evidencia de los efectos de su consumo, esta revisión revela que es necesaria más investigación de su uso en el ámbito deportivo.

\section{Palabras Clave:}

Mezquite, polifenoles, rendimiento deportivo, inflamación inducida por ejercicio.

\section{Introducción}

Los atletas de alto rendimiento (AAR) están constantemente sometidos a grandes cargas de esfuerzo físico, por lo que la nutrición es pilar para el mantenimiento de un buen estado de salud, además de desarrollar un papel clave en la mejora de la calidad de los entrenamientos y rendimiento en competencia. ${ }^{1}$

Los deportes de perfil intermitente alternan fases de esfuerzo de alta intensidad con periodos de recuperación variables, a diferencia de otros deportes que conllevan un esfuerzo físico de carácter continuo. Se pueden incluir en esta categoría deportes de equipo como el futbol, el baloncesto y el rugby, así como algunos individuales como el tenis y el bádminton. ${ }^{2}$ En estas condiciones, el consumo de oxígeno podría aumentar hasta 20 veces más que los valores en reposo. ${ }^{3}$ Por lo tanto, durante el ejercicio prolongado o de alta intensidad, donde la generación de especies reactivas de oxígeno (ROS) excede la capacidad antioxidante del cuerpo, es plausible la suplementación con antioxidantes como ayudas ergogénicas ( $\mathrm{AE})$, con el fin de contrarrestar la fatiga e incrementar el rendimiento. ${ }^{4}$ El término ergogénico se deriva del griego ergon (trabajo) y gennan (producir). Por lo tanto, ergogénico se refiere a cualquier estrategia que mejore la capacidad de trabajo. En el ámbito deportivo, las $A E$, son utilizadas para mejorar la producción de energía, el rendimiento deportivo (RD), la composición corporal y/ o potenciar las adaptaciones fisiológicas al entrenamiento. ${ }^{1}$

Recientemente se ha manifestado gran interés dirigido a las plantas y sus fitocomplejos; que proporcionan moléculas de interés para el RD. Entre los diversos fitoquímicos, los polifenoles (PF) representan una heterogénea clase de compuestos con marcadas propiedades antioxidantes, antiinflamatorias, antibacterianas, antivirales, antiparásitas y citotóxicas. ${ }^{5}$ Estos compuestos poseen la capacidad de eliminación de radicales gracias a su estructura química; los grupos hidróxilo pueden donar un electrón a los radicales y el anillo aromático puede estabilizar los radicales aroxilo resultantes. ${ }^{4}$

Se han realizado numerosos estudios acerca de la suplementación con PF, donde las fuentes principales suelen ser frutos rojos, como: cereza, zarzamora, arándano, y grosella, además de otras fuentes como la granada y la cocoa. ${ }^{3}$ En donde se ha demostrado su efecto al mejorar la tasa de recuperación, disminuir la inflamación y atenuar el dolor muscular. $3,4,6$

No obstante, existen otros recursos que poseen un significativo contenido de estos complejos moleculares; ejemplo de ello es el mezquite. Esta planta, perteneciente al género Prosopis, se encuentra dentro de la familia de las leguminosas, y se distribuye ampliamente en el territorio mexicano. Es considerado un recurso natural muy importante debido a sus diversos usos como alimento para ganado (hojas y vainas), alimentación humana en su presentación de harinas, bebidas fermentadas y en vainas $^{7}$. Se ha encontrado que los extractos obtenidos de cáscara, vainas, polen y hojas de plantas del mezquite, poseen compuestos fenólicos importantes como apigenina, catequina, juliflorina, quercetina, kaempfenol y mezquitol, además de una buena capacidad de eliminación de radicales libres. , $^{8,10,11}$

Sin embargo, la bibliografía e investigaciones del uso de esta planta en el ámbito deportivo es escasa, por lo que, el objetivo de esta revisión es conocer las propiedades y beneficios del mezquite, para evaluar su efecto sobre los fenómenos fisiológicos suscitados en deportes intermitentes y su factibilidad de uso como ayuda ergogénica. 


\section{Método}

Se realizó una revisión sistemática mediante búsqueda electrónica de artículos publicados en las plataformas PubMed, Google Académico y SciELO; dividiendo la búsqueda en tres temáticas principales: 1) Mezquite. Para la cual se utilizaron las palabras clave "Mesquite composition AND Mexico", "Mesquite AND polyphenols", "Mesquite AND exercise". Se incluyeron artículos tanto de revisión como experimentales, y se excluyeron aquellos cuyo estudio fuera hecho sobre especies fuera del territorio mexicano. 2) Polifenoles. La búsqueda fue realizada con las palabras clave "Polyphenols AND inflammation AND sport OR exercise", "Ergogenic aids AND polyphenols" incluyendo artículos originales y de revisión, sin discriminar el país de origen. 3) Inflamación inducida por ejercicio. Utilizando "Sport performance AND intermittent sport AND inflammation", "Intermittent exercise AND muscle inflammation AND performance AND damage" como palabras clave. De igual manera, se incluyeron artículos originales, revisiones y tesis de cualquier país de origen.

En los tres casos se incluyeron únicamente artículos publicados entre el 2015 y 2020 - a excepción de los artículos de "Mezquite" donde se tomaron en cuenta artículos desde el año 2010-. Procurando que al menos el $80 \%$ de los mismos fueran en idioma inglés.

Los artículos fueron seleccionados manualmente de acuerdo a su relevancia en el perfil de esta revisión.

\section{Resultados}

Se localizaron 33 artículos (11 de "Mezquite", 10 de "Polifenoles", y 12 de "Inflamación inducida por ejercicio"), pero después de una revisión exhaustiva, fueron descartados 17 de ellos, por no cumplir con los criterios de inclusión, o tener poca relevancia; resultando un total de 24 artículos (Tabla 1).

Tabla 1. Resultados de la búsqueda y selección de artículos.

\begin{tabular}{cccccc}
\hline Temática & $\begin{array}{c}1^{\circ} \text { Revisión } \\
\text { (artículos) }\end{array}$ & $\begin{array}{c}2^{\circ} \text { Revisión } \\
\text { (artículos) }\end{array}$ & $\mathrm{E}^{*}$ & $\mathrm{R}^{*}$ \\
\hline Mezquite & 11 & 5 & 5 & 0 \\
\hline Polifenoles & 10 & 7 & 3 & 4 \\
\hline $\begin{array}{c}\text { Inflamación } \\
\text { inducida por } \\
\text { ejercicio. }\end{array}$ & 12 & 4 & 1 & 2 \\
\hline TOTAL & 33 & 16 & 9 & 7 \\
\hline
\end{tabular}

${ }^{*} \mathrm{E}$ : artículos experimentales; R: artículos de revisión.

\section{Mezquite}

Los artículos encontrados sobre "Mezquite", fueron en su totalidad, de tipo experimental; donde se evaluó su composición, contenido de compuestos bioactivos y propiedades de interés para la industria alimentaria y el consumo humano. Dichos análisis fueron realizados sobre distintas fracciones del mezquite; lo cual arroja resultados variables de interés sobre el contenido y propiedades de cada una de ellas. En la Tabla 2 se enlistan aquellos de mayor relevancia con especial atención en sus compuestos fenólicos.

Todos los estudios presentaron datos de composición química proximal (AQP) a excepción del primero ${ }^{8}$, el cual tampoco presentó cifras concretas del contenido de fenoles, flavonoides y actividad antirradical 1,1-difenil-2picrilhidrazil (DPPH).

En aquellos artículos donde se evaluaron varias fracciones del mezquite sometidas a distintos tratamientos, ${ }^{10,12,13}$ se eligieron aquellas que presentaron mayor contenido de compuestos bioactivos, o bien, mayor ventaja en cuanto a composición sobre el resto de las fracciones, presentando datos tanto de la muestra original, como de la tratada.

En todos los artículos, se reportó contenido de fenoles, flavonoides y actividad antirradical DPPH, excepto en el de Ibarra y cols. (2017) $)^{14}$ y Gallegos cols. $(2013)^{13}$ el cual sólo mencionó el contenido de fenoles totales.

Adicionalmente, dos de los estudios, ${ }^{10,12}$ proporcionan el perfil de aminoácidos de las fracciones evaluadas.

\section{Polifenoles (PF)}

Fueron identificados 4 artículos de revisión en los cuales se mencionan los principales compuestos fenólicos hallados y las fuentes dietéticas de los mismos, así como los efectos reportados sobre el RD tras su consumo. Fueron de especial interés aquellos compuestos que fueron reconocidos en los artículos de composición de mezquite. La Tabla 3 enlista los resultados obtenidos de los estudios realizados sobre dichos compuestos en común.

A pesar de que se ha demostrado el efecto positivo que tienen estos compuestos sobre el RD, no se ha encontrado un estudio donde se analicen dichos compuestos, utilizando el mezquite como fuente. 
Tabla 2. Composición e identificación de compuestos bioactivos en el mezquite (Prosopis spp.)

\begin{tabular}{|c|c|c|}
\hline Referencia & Prosopis planta y fracción & Hallazgos principales \\
\hline $\begin{array}{l}\text { Ramírez, Vargas, } \\
\text { Hernández, Martínez, } \\
\text { Sánchez, Torrescano, } \\
\text { y Sánchez }(2020)^{9} .\end{array}$ & $\begin{array}{l}\text { Prosopis velutina } \\
\text { Hoja }\end{array}$ & $\begin{array}{l}\text { Fenoles totales } \\
>75 \mathrm{mg} \mathrm{EAG*/g} \\
\text { Flavonoides totales } \\
>100 \mathrm{mg} \mathrm{ER} / \mathrm{g} \\
\text { Actividad antiradical DPPH } \\
>25 \% \text { inhibición }\end{array}$ \\
\hline $\begin{array}{l}\text { Díaz, } \quad \text { Hernández, } \\
\text { Román, } \\
\text { Castro, } \quad \text { Cariño, } \\
\text { Gómez }(2018)^{10} \text {. y }\end{array}$ & $\begin{array}{l}\text { Prosopis laegivata } \\
\text { Harina de mesocarpio y semilla. } \\
\text { En sus modalidades: } \\
\text { Cruda (MSF) } \\
\text { Térmicamente tratada (MSFT), e } \\
\text { Hidrolizada (MSFH) }\end{array}$ & $\begin{array}{l}\text { AQP MSF } \\
\text { Proteína } 11.93 \mathrm{~g} / 100 \mathrm{~g} \\
\text { Humedad } 7.71 \mathrm{~g} / 100 \mathrm{~g} \\
\text { Fibra cruda } 4.2 \mathrm{~g} / 100 \mathrm{~g} \\
\text { Grasa } 1.81 \mathrm{~g} / 100 \mathrm{~g} \\
\text { Cenizas } 4.07 \mathrm{~g} / 100 \mathrm{~g} \\
\text { Fenoles totales } \\
\text { MSF } 8.36 \mathrm{mg} \mathrm{EAG*} / \mathrm{g} \\
\text { MSFT } 13.2 \mathrm{mg} \mathrm{EAG*/g} \\
\text { Flavonoides totales } \\
\text { MSF } 1.80 \mathrm{mg} / \mathrm{g}(0.30 \mathrm{mg} \mathrm{QE} / \mathrm{g}+1.50 \mathrm{mg} \mathrm{CE} / \mathrm{g}) \\
\text { MSFT } 1.32 \mathrm{mg} / \mathrm{g}(0.59 \mathrm{mg} \mathrm{QE} * / \mathrm{g}+0.73 \mathrm{mg} \mathrm{CE} / \mathrm{g}) \\
\text { MSFH } 5.27 \mathrm{mg} / \mathrm{g}\left(2.63 \mathrm{mg} \mathrm{QE} / \mathrm{g}+2.64 \mathrm{mg} \mathrm{CE}^{*} / \mathrm{g}\right) \\
\text { Actividad antirradical DPPH } \\
\text { MSF } 10.1 \mathrm{mg} \mathrm{AAE*} / \mathrm{g} \\
\text { MSFT } 17.9 \mathrm{mg} \mathrm{AAE} / \mathrm{g}\end{array}$ \\
\hline $\begin{array}{l}\text { Díaz, } \\
\text { Gutiérrez, } \\
\text { Castro, } \quad \text { Pérezállez, } \\
\text { Gómez }(2018)^{12} \text {. }\end{array}$ & $\begin{array}{l}\text { Prosopis laegivata } \\
\text { Harina de semillas } \\
\text { Cruda (SF) } \\
\text { Extruida (SFE) }\end{array}$ & $\begin{array}{l}\text { AQP SF } \\
\text { Extracto libre de nitrógeno } 38.45 \mathrm{~g} / 100 \mathrm{~g} \\
\text { Proteína } 36.51 \mathrm{~g} / 100 \mathrm{~g} \\
\text { Humedad } 8.28 \mathrm{~g} / 100 \mathrm{~g} \\
\text { Fibra cruda } 7.73 \mathrm{~g} / 100 \mathrm{~g} \\
\text { Grasa } 4.83 \mathrm{~g} / 100 \mathrm{~g} \\
\text { Cenizas } 4.14 \mathrm{~g} / 100 \mathrm{~g} \\
\text { Fenoles totales } \\
\text { SF } 6.68 \mathrm{mg} \text { EAG*/g } \\
\text { SFE } 6.46 \mathrm{mg} \mathrm{EAG*} / \mathrm{g} \\
\text { Flavonoides totales } \\
\text { SF } 0.0416 \mathrm{mg} \mathrm{Apigenina} / \mathrm{g} \\
\text { SFE } 0.0395 \mathrm{mg} \mathrm{Apigenina/g} \\
\text { Actividad antirradical DPPH } \\
\text { SF } 9.11 \mathrm{mg} \text { AAE*/g } \\
\text { SFE } 9.32 \mathrm{mg} \mathrm{AAE*/g}\end{array}$ \\
\hline
\end{tabular}




\begin{tabular}{|c|c|c|}
\hline $\begin{array}{l}\text { lbarra, Castañón, } \\
\text { Martínez, Ávila y Ayala } \\
(2017)^{14} \text {. }\end{array}$ & $\begin{array}{l}\text { Prosopis spp. } \\
\text { Vaina completa }\end{array}$ & $\begin{array}{l}\text { AQP } \\
\text { Extracto libre de nitrógeno } 20.9 \mathrm{~g} / 100 \mathrm{~g} \\
\text { Proteína } 11.4 \mathrm{~g} / 100 \mathrm{~g} \\
\text { Humedad } 26.9 \mathrm{~g} / 100 \mathrm{~g} \\
\text { Fibra cruda } 23.5 \mathrm{~g} / 100 \mathrm{~g} \\
\text { Grasa } 1.1 \mathrm{~g} / 100 \mathrm{~g} \\
\text { Cenizas } 16.2 \mathrm{~g} / 100 \mathrm{~g}\end{array}$ \\
\hline $\begin{array}{l}\text { Gallegos, } \\
\text { González yocha, } \\
(2013)^{13}\end{array}$ & $\begin{array}{l}\text { Prosopis laevigata } \\
\text { Harina de mesocarpio y semilla } \\
\text { (MSF) } \\
\text { Harina de mesocarpio y semilla } \\
\text { con tratamiento térmico (MSFT) }\end{array}$ & $\begin{array}{l}\text { AQP MSF } \\
\text { Extracto libre de nitrógeno } 65.39 \mathrm{~g} / 100 \mathrm{~g} \\
\text { Proteína } 22.51 \mathrm{~g} / 100 \mathrm{~g} \\
\text { Humedad } 9.82 \mathrm{~g} / 100 \mathrm{~g} \\
\text { Fibra cruda } 2.38 \mathrm{~g} / 100 \mathrm{~g} \\
\text { Grasa } 3.35 \mathrm{~g} / 100 \mathrm{~g} \\
\text { Cenizas } 5.83 \mathrm{~g} / 100 \mathrm{~g} \\
\text { AQP MSFT } \\
\text { Extracto libre de nitrógeno } 69.13 \mathrm{~g} / 100 \mathrm{~g} \\
\text { Proteína } 20.97 \mathrm{~g} / 100 \mathrm{~g} \\
\text { Humedad } 3.06 \mathrm{~g} / 100 \mathrm{~g} \\
\text { Fibra cruda } 2.41 \mathrm{~g} / 100 \mathrm{~g} \\
\text { Grasa } 2.08 \mathrm{~g} / 100 \mathrm{~g} \\
\text { Cenizas } 5.37 \mathrm{~g} / 100 \mathrm{~g} \\
\text { Fenoles totales } \\
\text { MSF } 22.46 \mathrm{mg} \text { EAG*/g } \\
\text { MSFT } 19.02 \mathrm{mg} \mathrm{EAG*/g}\end{array}$ \\
\hline
\end{tabular}

"EAG: Equivalentes de ácido gálico; ER: Equivalentes de rutina; QE: Equivalentes de quercetina; CE: Equivalentes de catequina; AAE: Equivalentes de ácido ascórbico.

Tabla 3. Compuestos fenólicos contenidos en el mezquite, fuentes adicionales, y efectos reportados tras su aplicación en población deportista.

\begin{tabular}{|c|c|c|}
\hline Compuesto & Efectos reportados & Fuentes \\
\hline Apigenina & $\begin{array}{l}\text { Modulación del estrés oxidativo, función hormonal, metabolismo energético, } \\
\text { expresión génica y proceso epigenético }{ }^{12} \text {. }\end{array}$ & Mezquite $^{12}$. \\
\hline Catequina & $\begin{array}{l}\text { Reducción de la magnitud de daño muscular, estrés oxidativo en respuesta a } \\
\text { la fatiga, y tiempo de recuperación del ejercicio }{ }^{5} \text {. } \\
\text { Aumento de } \mathrm{VO}_{2} \text { máx. debido a una mayor extracción de oxígeno en el } \\
\text { músculo ejercitado, con una mejor distribución espacial de perfusión } \\
\text { muscular }{ }^{4} \text {. } \\
\text { Modulación del estrés oxidativo y proceso inflamatorio }{ }^{5} \text {. }\end{array}$ & $\begin{array}{l}\text { Té verde }^{4,5} \text {. } \\
\text { Cacaco }^{5} \text {. } \\
\text { Mezquite }^{9,10} \text {. }\end{array}$ \\
\hline Quercetina & $\begin{array}{l}\text { Mejora del rendimiento durante el ejercicio prolongado; incrementando el } \mathrm{VO}_{2} \\
\text { max y tiempo de resistencia a la fatiga }{ }^{15} \text {. } \\
\text { Inhibición de la xantina oxidasa y NADPH oxidasa (productoras de especies } \\
\text { reactivas de oxígeno). } \\
\text { Atenuación del dolor y el daño muscular inducido por ejercicio, calambres y } \\
\text { aceleración de la recuperación muscular }{ }^{5,16} \text {. }\end{array}$ & $\begin{array}{l}\text { Cebolla }^{16} . \\
\text { Arándano }^{16} \text {. } \\
\text { Col rizada }^{16} \text {. } \\
\text { Manzana }^{16} . \\
\text { Zarzamora }^{17} \text {. } \\
\text { Mango }^{16} . \\
\text { Mezquite }^{9,10,12} \text {. }\end{array}$ \\
\hline Luteolina & Mejora del rendimiento del ejercicio de velocidad ${ }^{16}$. & $\begin{array}{l}\text { Perejilit } \\
\text { Mezquite }^{12}\end{array}$ \\
\hline
\end{tabular}


La Tabla 4 resume los hallazgos obtenidos de los 3 artículos experimentales, detallando las características de la población de estudio, las pruebas físicas, el protocolo de suplementación, los indicadores evaluados, y los efectos encontrados.

En los estudios de Bell y cols. (2016) ${ }^{6}$ y García y cols. (2017), ${ }^{17}$ se trabajó con atletas de un deporte definido, mientras que, en el caso de Martín y cols. (2020), ${ }^{16}$ la naturaleza de la muestra fue más heterogénea.

Las pruebas físicas a las cuales se sometieron los atletas fueron previamente planeadas y controladas para evaluar los indicadores de rendimiento, dentro de dichas pruebas, el salto con contramovimiento (CMJ) y dolor muscular de inicio retardado (DOMS) fueron tomadas en cuenta por dos de los estudios. ${ }^{6,16}$ No obstante, en el caso de García y cols. (2017), ${ }^{17}$ la prueba física consistió en la competencia misma, lo cual sugeriría un nivel de esfuerzo sumamente variable entre los competidores.

Los tres estudios tuvieron en común la evaluación de Creatin quinasa (CK) como marcador de daño muscular e inflamación.

\section{Inflamación inducida por ejercicio}

Finalmente, de "inflamación inducida por ejercicio" se halló 1 artículo experimental y 3 de revisión, de los cuales se destacan las principales células y mecanismos moleculares afectados por el ejercicio.

\section{Discusión}

\section{Mezquite}

Se identificó un mayor contenido de fenoles totales en el extracto de hoja de $P$. velutina, ${ }^{9}$ así como en la fracción MSF de $P$. laegivata estudiada por Gallegos (2013). ${ }^{13} \mathrm{Se}$ demostró, además, que durante los procesos térmicos aplicados a las harinas puede prevenirse la pérdida de fenoles al manejar una relación temperatura tiempo adecuada, puesto que, al someter las harinas a altas temperaturas por un periodo prolongado de tiempo, se reduce la estabilidad de los fenoles, provocando la destrucción de algunos de ellos. Sin embargo, en periodos más cortos de tiempo, esto puede resultar beneficioso, pues es posible incrementar la fracción de ácidos fenólicos libres y su redistribución, a consecuencia de la ruptura de los fenoles unidos a otros compuestos como los esterificados y los glicosilados. ${ }^{13}$ Para fines del uso del mezquite como $\mathrm{AE}$, es esencial prestar especial atención a los procesos tecnológicos a los que se le someta, pues la finalidad es conservar su valor nutricional funcional.
El mayor contenido de flavonoides fue el reportado en el extracto de hoja de $P$. Velutina, seguido de la MSF de $P$. laegivata estudiada por Díaz y cols. (2018), la cual, tras un proceso de hidrólisis ácida, mostró un aumento del 180\% en su contenido de flavonoides. ${ }^{10}$ Este hecho conduce a considerar la hidrólisis como un proceso tecnológico viable para acentuar la calidad nutrimental del mezquite. La MSFT de este mismo estudio presentó la mayor actividad antirradical DPPH a consecuencia del tratamiento térmico, efecto también experimentado por la SFT. ${ }^{12}$ Este indicador es altamente relevante para la presente revisión, puesto que indica el poder antioxidante del producto, es decir, la capacidad de inhibición de radicales libres generados por los AAR durante el ejercicio.

Otra de las ventajas que presentan las fracciones MSF reportada por Díaz y cols. $(2018)^{10}$ y la MSFT de Gallegos y cols. (2013), ${ }^{13}$ es su contenido de grasa, el cual, de acuerdo al CODEX CAC/GL 23-1997 puede declararse como "bajo" al no superar los $3 \mathrm{~g}$ por $100 \mathrm{~g}$ de producto. ${ }^{18}$ Finalmente, se destaca la $\mathrm{SF}^{12}$ como la fracción con más alto contenido proteico, además de un perfil de aminoácidos de alto valor, con valina como único aminoácido limitante. A pesar de que el presente trabajo, se centra en el contenido de compuestos fenólicos del mezquite como propuesta de $\mathrm{AE}$, estos resultados dejan abierta una línea de investigación adicional.

\section{Polifenoles (PF)}

El impacto de la suplementación con PF se ve reflejado en la reducción del estrés oxidativo en respuesta a la fatiga gracias a la presencia de compuestos como apigenina, catequina y quercetina; mismos que pueden encontrarse en el mezquite (Tabla 3).

En el estudio realizado con extracto de hoja de mango (Tabla 4), se atribuye este efecto a la inhibición de las enzimas productoras de ROS (NADPH oxidasa y xantina oxidasa), así como la capacidad antirradical del extracto. Esto sugiere que el mezquite -específicamente la MSFT de $P$. laegivata ${ }^{10}$ - podría ser útil en la neutralización de dichos compuestos, gracias a su alta actividad radical reportada (Tabla 2 ).

Se ha demostrado que los radicales libres son responsables de la nocicepción, ${ }^{16}$ causa principal del DOMS, lo que merma el RD. Sin embargo, tras la suplementación con PF, en los estudios de Bell y cols. $(2016)^{6}$ y Martín y cols. $(2020)^{16}$ se redujo dicho indicador; se presume que la quercetina es la responsable de este hecho, puesto que se ha probado anteriormente su efecto en la reducción de la nocicepción, atenuando el dolor experimentado por los atletas. ${ }^{16}$ 
Tabla 4. Efectos de la suplementación con polifenoles en el rendimiento deportivo.

\begin{tabular}{|c|c|c|c|c|c|}
\hline Referencia & $\begin{array}{l}\text { Características de la } \\
\text { población de estudio }\end{array}$ & $\begin{array}{l}\text { Pruebas } \\
\text { físicas }\end{array}$ & $\begin{array}{l}\text { Protocolo de } \\
\text { suplementación }\end{array}$ & $\begin{array}{l}\text { Indicadores } \\
\text { evaluados }\end{array}$ & Resultados \\
\hline $\begin{array}{l}\text { Bell y cols. } \\
(2016)^{6} \text {. }\end{array}$ & $\begin{array}{l}16 \text { jugadores } \\
\text { masculinos de futbol } 25 \\
\pm 4 \text { años con al menos } \\
3 \text { años de } \\
\text { entrenamiento } \\
\text { consistente } \\
\text { consecutivo } \\
\text { Se dividieron } \\
\text { aleatoriamente en dos } \\
\text { grupos iguales: } \\
\text { experimental y placebo } \\
\text { (Diseño doble ciego } \\
\text { controlado por placebo) }\end{array}$ & $\begin{array}{l}\text { Tiempo de } \\
\text { sprint } 20 \text { m } \\
\text { Test de agilidad } \\
5-0-5 \\
\text { CMJ } \\
\text { Contracción } \\
\text { isométrica } \\
\text { voluntaria } \\
\text { máxima (MVIC) } \\
\text { DOMS }\end{array}$ & $\begin{array}{l}\text { Concentrado de Cereza } \\
\text { Montmorency } \\
30 \text { ml, } 2 \text { veces al día, por } \\
7 \text { días consecutivos } \\
\text { ( } \text { días antes, y los } 3 \\
\text { días de prueba) } \\
\text { Composición: } \\
\text { Antocianinas totales: } \\
73.5 \mathrm{mg} \mathrm{L}^{-1} \\
\text { de cianidina } \\
\text { Fenoles totales: } 178.8 \\
\text { eq. De ácido gálico } \mathrm{L}^{1}\end{array}$ & $\begin{array}{l}\mathrm{CK}^{*} \\
\text { IL-1- } \beta^{*} \\
\text { IL-6* } \\
\text { TNF-- } \\
\text { hsCRP* } \\
\text { LOOH }^{*}\end{array}$ & $\begin{array}{l}\text { Los índices de } \\
\text { rendimiento } \\
\text { (CMJ, MVIC y } \\
\text { agilidad) se } \\
\text { recuperaron más } \\
\text { rápido, y la } \\
\text { calificación de } \\
\text { DOMS fue más } \\
\text { baja en el grupo } \\
\text { suplementado. } \\
\text { Atenuación de la } \\
\text { respuesta } \\
\text { inflamatoria } \\
\text { aguda (IL-6). }\end{array}$ \\
\hline $\begin{array}{l}\text { Martín y } \\
\text { cols. } \\
(2020)^{16} \text {. }\end{array}$ & $\begin{array}{l}48 \text { sujetos de estudio } \\
(18 \mathrm{M} \text { y } 30 \mathrm{H}) \text {, de entre } \\
18-45 \quad \text { años, } \\
\text { físicamente activos y } \\
\text { ejercitados participantes } \\
\text { regularmente. } \\
\text { Los asignados } \\
\text { fueron an o } \\
\text { aleatoriamente a un } \\
\text { grupo de placebo o } \\
\text { polifenoles. Ambos } \\
\text { grupos se equipararon } \\
\text { por tiempos de carrera } \\
\text { en una prueba previa } \\
\text { de } 5 \mathrm{~km} \text {, así como por } \\
\text { sexo. }\end{array}$ & $\begin{array}{l}\text { Carrera de } 10 \\
\text { km } \\
\text { Seguida por } \\
100 \text { saltos de } \\
\text { caída desde un } \\
\text { escalón de } 59 \\
\text { cm de altura }(5 \\
\text { series de } 20 \\
\text { repeticiones } \\
\text { con un periodo } \\
\text { de } \\
\text { recuperación } \\
\text { de } 2 \text { minutos } \\
\text { entre serie) } \\
\text { CMJ } \\
\text { DOMS }\end{array}$ & $\begin{array}{l}\text { Consumo de } 2 \\
\text { cápsulas, cada una con } \\
70 \text { mg de Zinamita } \\
\text { (extracto de hoja de } \\
\text { mangifera indica } \\
\text { estandarizado a } 60 \% \text { de } \\
\text { manguiferina) } \\
\text { combinado con } 70 \mathrm{mg} \\
\text { de quercetina y } 153 \mathrm{mg} \\
\text { de maltodextrina. } \\
1 \text { hora antes de la } \\
\text { competencia y cada } 8 \\
\text { horas después por } 24 \\
\text { horas. }\end{array}$ & $\begin{array}{l}\text { Lactato en } \\
\text { sangre } \\
\text { Mioglobina } \\
\text { hsCRP* } \\
\text { ALT* }^{*} \\
\text { CK }^{*}\end{array}$ & $\begin{array}{l}\text { Disminución del } \\
\text { dolor, daño } \\
\text { muscular y } \\
\text { tiempo de } \\
\text { recuperación } \\
\text { tras carrera de } \\
10 \mathrm{k} \\
\text { Atenuación de la } \\
\text { pérdida de } \\
\text { rendimiento en } \\
\text { CMJ } \\
\text { Atenuación del } \\
\text { aumento de } \\
\text { mioglobina y ALT } \\
\text { en hombres. }\end{array}$ \\
\hline $\begin{array}{l}\text { García y } \\
\text { cols } \\
(2017)^{17} \text {. }\end{array}$ & $\begin{array}{l}14 \quad \text { atletas de } \\
\text { balonmano con } \\
\text { experiencia en torneos } \\
\text { nacionales } \\
\text { internacionales. } \\
\text { Selección aleatoria en } \\
\text { grupos iguales: } \\
\text { experimental y placebo. }\end{array}$ & $\begin{array}{l}\text { Competencia } \\
\text { nacional oficial } \\
\text { de balonmano } \\
\text { con duración de } \\
6 \text { días }\end{array}$ & $\begin{array}{l}200 \mathrm{~g} \text { de zarzamora } \\
\text { congelada } \\
\text { homogenizada en } 200 \\
\mathrm{ml} \text { de agua con } \\
\text { contenido de } 11.1 \mathrm{~g} \text { de } \\
\text { fenoles totales y } 13.8 \\
\mathrm{mM} \text { de actividad } \\
\text { antioxidante por } 100 \mathrm{gr} \\
\text { de fruta. } \\
\text { Administración antes del } \\
\text { almuerzo por } 16 \text { días, } \\
\text { iniciando una semana } \\
\text { previa a la competencia, } \\
\text { durante los } 6 \text { días de } \\
\text { competencia y } 3 \text { días } \\
\text { después. }\end{array}$ & $\begin{array}{l}\mathrm{CK}^{*} \\
\text { Urea }\end{array}$ & $\begin{array}{l}\text { Tendencia a una } \\
\text { recuperación } \\
\text { más rápida de } \\
\text { los valores de } \\
\mathrm{CK} \text { a las } 24 \mathrm{~h} \text { en } \\
\text { el grupo } \\
\text { experimental }\end{array}$ \\
\hline
\end{tabular}

${ }^{*}$ CK: Creatin kinasa; IL-1: Interleucina-1-beta; IL-6: Interleucina-6; TNAF-a: Factor de necrosis tumoral alfa; hsCRP: Proteína C reactiva de alta sensibilidad; LOOH: Hidroperóxidos lipídicos; ALT: Alanina aminotransferasa sérica. 
La quercetina, es el compuesto fenólico del cual se reportó la mayor cantidad de beneficios sobre el RD (Tabla 3). Esto se relaciona con su capacidad de interferir con los mecanismos inflamatorios, reduciendo la producción del factor de necrosis tumoral alfa (TNF-a) e interleucinas; esto puede observarse claramente en los resultados de los estudios de suplementación con cereza ${ }^{6}$ y zarzamora ${ }^{17}$ al resultar en una atenuación en los niveles de II-6 y CK respectivamente, con tendencia en ambos estudios a un menor tiempo de recuperación de los atletas, debido a la alta capacidad antioxidante de la quercetina, que es 5 veces mayor que las vitaminas $C$ y E. ${ }^{17}$ El mezquite es rico en este compuesto, presentando sus mayores niveles en la MSFH de $P$. laegivata ${ }^{10}$ (Tabla 4).

Aunado a la evidencia existente de los efectos positivos de los compuestos encontrados en el mezquite sobre el rendimiento en deportes de perfil intermitente, éste se convierte en un candidato potencial para ser utilizado como ingrediente o fuente principal de PF en AE's destinadas a AAR.

\section{Inflamación inducida por ejercicio}

Una de las condiciones mayormente experimentadas por los AAR, son las infecciones del tracto respiratorio superior (URTI) debido a la teoría de "ventana abierta" que consiste en un momento determinado con un alto grado de inmunosupresión, donde los atletas son más propensos a contraer infecciones. ${ }^{19}$

Como consecuencia de esta inmunosupresión, también se presenta un fenómeno evidente de inflamación. Las células que están implicadas en los procesos de inflamación inducida por ejercicio, y que frecuentemente son utilizadas como biomarcadores en diversos estudios son NK, neutrófilos, macrófagos, células dendríticas, linfocitos y citocinas (principalmente IL-6, IL1, IL-8, TNF-a). ${ }^{20,21}$

Aunque se sabe que con el entrenamiento se desarrollan adaptaciones fisiológicas ante los altos volúmenes de esfuerzo, muchas veces se supera la capacidad fisiológica del atleta. Se ha comprobado que ciertos suplementos dietéticos pueden estimular la función inmunológica y reducir la incidencia de URTI, en especial los PF como la quercetina. ${ }^{22}$ Es aquí donde entra en juego el mezquite, pues dado su contenido y perfil de PF, y su acentuada actividad antirradical, es capaz de disminuir la expresión de células inflamatorias y pro inflamatorias, lo que además de favorecer el $\mathrm{RD}$, proporciona una función profiláctica significativa.

\section{Conclusiones}

El mezquite es un recurso natural potencialmente rentable para ser utilizado como ayuda ergogénica. La harina de mesocarpio y la semilla del mezquite poseen un importante contenido de polifenoles y capacidad antioxidante, además de un buen perfil de aminoácidos y un bajo contenido de grasa. Los compuestos bioactivos encontrados en el mezquite favorecen la reducción del estrés oxidativo, la atenuación del dolor muscular, y la disminución del tiempo de recuperación post ejercicio.

En vista de todos los beneficios atribuidos a la composición del mezquite, pero a la escasa evidencia de los efectos de su consumo, esta revisión revela que es necesaria más investigación de su uso en el ámbito deportivo. Desde un punto de vista nutricional, el estudio y aplicación de compuestos funcionales de fuentes naturales, así como el desarrollo de nuevos productos basados en recursos abundantes en el territorio nacional, significa una ventaja para la nutrición deportiva en la población mexicana, debido a su bajo coste y accesibilidad.

\section{Conflicto de intereses}

Los autores declaramos que no tenemos conflicto de intereses.

\section{Referencias}

[1] Bagchi, D., Nair, S., \& Sen, C. (2019). Nutrition and enhanced sports performance: Muscle building, endurance, and strength. 2nd ed. United States: Elsevier

[2] Monteaguado. P. (2016). El entrenamiento intermitente de alta intensidad (EIAI) como herramienta en la mejora del rendimiento en deportes de perfil intermitente. España: Universidad Miguel Hernández. Pp. 1-12.

[3] Massaro, M., Scoditti, E., Carluccio, M.A., Kaltsatou, A., \& Cicchella, A. (2019). Effect of Cocoa Products and Its Polyphenolic Constituents on Exercise Performance and Exercise-Induced Muscle Damage and Inflammation: A Review of Clinical Trials. Nutrients, 11(7), 14711486.

[4] Bowtell, J., \& Kelly, V. (2019). Fruit-Derived Polyphenol Supplementation for Athlete Recovery and Performance. Sports Medicine, 49(1), 3-23.

[5] Sorrenti, V., Fortinguerra, S., Caudullo, G., \& Buriani, A. (2020). Deciphering the Role of Polyphenols in Sports Performance: From Nutritional Genomics to the Gut Microbiota toward Phytonutritional Epigenomics. (2020). Nutrients, 12(5), 1265-1293.

[6] Bell, P., Stevenson, E., Davidson, G., \& Howatson, G. (2016). The Effects of Montmorency Tart Cherry Concentrate Supplementation on Recovery Following Prolonged, Intermittent Exercise. Nutrients, 8(7), 441-452.

[7] Comisión Nacional Forestal. (2015). Prosopis laegivata. Recuperado de http://www.conafor.gob.mx:8080/documentos/docs/13/988Prosopis\% 20laevigata.pdf Fecha de acceso: 15 de septiembre de 2020.

[8] Sharifi, J., Kobarfard, F., Ata, A., Abdulmajid, S., Khosravi, N., Kumar, A., Tomas, M., Capanoglu, E., Matthews, K., Popovic, J., Kostic, A., 
Kamiloglu, S., Sharopov, F., Iqbal, M., \& Martins, N. (2019). Prosopis Plant Chemical Composition and Pharmacological Attributes: Targeting Clinical Studies from Preclinical Evidence. Biomolecules, 9(12), 777--823.

[9] Ramírez, R.M., Vargas, S.R., Hernández, M.J., Martínez, B.E., Sánchez, E.J., Torrescano, U.G., \& Sánchez, E.A. (2019). Actividad antioxidante de extractos de hoja de mezquite (Prosopis velutina). Biotecnia, 21(1), 113-119.

[10] Díaz, L., Hernández, J., Román, A., Cariño, R., Castro, J., Téllez, A. \& Gómez, C. (2018). Chemical and nutritional characterization of raw and thermal-treated flours of Mesquite (Prosopis laevigata) pods and their residual brans. Journal of Food, 16(1), 444-451.

[11] Cattaneo, F., Costamagna, M. S., Zampini, I. C., Sayago, J., Alberto, M. R., Chamorro, V., \& Isla, M. I. (2016). Flour from Prosopis alba cotyledons: A natural source of nutrient and bioactive phytochemicals. Food Chemistry, 208, 89-96.

[12] Díaz, L., Hernández, J., Gutiérrez, R., Téllez, A., Castro, J., Pérez, R., \& Gómez, C. (2018). Nutritional Characterization of Prosopis laevigata Legume Tree (Mesquite) Seed Flour and the Effect of Extrusion Cooking on its Bioactive Components. Foods, 7(124), 1-9.

[13] Gallegos, J., Rocha, N., González, R., \& García, M. (2013). Efecto del procesamiento térmico sobre la capacidad antioxidante de pinole a base de vainas de mezquite (Prosopis laevigata). CyTA Journal of Food. 11(2), 162-170.

[14] Ibarra, M., Castañón, J., Martínez, M., Ávila, L., \& Ayala, I. (2017). Análisis de las propiedades de la harina de mezquite para el uso como ayuda ergogénica. Revista de Ciencias del Ejercicio FOD. 11(2), 1-12.

[15] de León, G., Rodríguez, J., Candia, R., Carrasco, C., \& Enriquez, L. (2019). Efectividad de los suplementos antioxidantes en la mejoría del desempeño físico atlético. Artículo de revisión. Revista Habanera de Ciencias Médicas. 18(2), 192-216.

[16] Martín, M., Gelabert, M., Galván, V., Gallego, A., Martínez, M., López, L. Wiebe, J., Martín, S., Arteaga, R., Dorado, C., Pérez, S., Santana, A., Morales, D., \& Calbet, J. (2020). Supplementation with a Mango Leaf Extract (Zynamite $®)$ in Combination with Quercetin Attenuates Muscle Damage and Pain and Accelerates Recovery after Strenuous Damaging Exercise. Nutrients. 12(614), 1-15.

[17] García, M., Gutiérrez, G., Estrada, S., González, B., Rodríguez, E., \& Rangel, B. (2017). Protección antioxidante de zarzamora para disminuir daño muscular en atletas de elite. Revista de psicología del deporte. 26(2), 157-163.

[18] Organización de las Naciones Unidas para la Alimentación y la Agricultura. (2001). Directrices para el uso de declaraciones nutricionales y saludables CAC/GL 23-1997. Recuperado de http://www.fao.org/ag/humannutrition/33313033ebb12db9b719ac1c14f821f5ac8e36.pdf Fecha de acceso 25/09/20.

[19] Alack, K., Pilat, C., \& Krüger, K. (2019). Current knowledge and new challenges in excersice immunology. German Journal of Sports Medicine, 70(10), 250-260.

[20] Kostrzewa, D., Ciechanowicz, A., Clark, J., \& Nowak, R. (2020). Damage-associated molecular patterns and Th-cell-cytokines released after progressive effort. Journal of Clinical Medicine. 9, 1-13.

[21] Eguasi, S., Pushpamithran, G., Richter, P., \& Attakora, K. (2017). Exercise Immunology: Involved Components and Varieties in Different Types of Physical Exercise. ScientecJournal of Life Sciences. $1(1), 31-35$.

[22] Gleeson, M. (2015). Immunological aspects of sport nutrition. Immunology and Cell Biology, 94(2), 117-123 